\title{
Detection of Pathogenic Viruses in the Ambient Air in Seoul, Korea
}

Tae-Hee Han'1, Sang-Hun Park², Ju-Young Chung³ ${ }^{3}$ Hyo-Won Jeong², Jihun Jung², Jae-In Lee², Young-Ok Hwang², II-Young Kim², Jip-Ho Lee², Kweon Jung²

${ }^{1}$ Department of Diagnostic Laboratory Medicine and ${ }^{3}$ Department of Pediatrics, SanggyePaik Hospital, Inje University College of Medicine, Seoul, Korea

2Department of Microbiology, Seoul Metropolitan Environmental Health Institute, Seoul, Korea

\section{Objectives}

The purpose of the present study was to detect pathogenic viruses in the ambient air in Seoul during the Asian and non-Asian dust periods

\section{Methods}

- Air samples were collected at three meteorological observation station located in the southern, eastern, and northern parts of Seoul.

- Between March and September 2016, ambient air samples were collected at flow rates of 6-8 $\mathrm{L} /$ min using polycarbonate (PC) filters (SKC Inc Eighty Four, PA) with pore sizes of $0.4 \mu \mathrm{m}$ for 4 hours (Verreault et al. 2010).

- Information about Asian dust was obtained from the open access database of the Korean Meteorological association (http://web.kma.go.kr/info_open/public_data/guid epage).

- RT-PCR was performed to detect respiratory viral pathogens [Influenza A (IF-A) and IF-B, respiratory syncytial virus (RSV)-A and RSV-B, human adenoviruses, human metapneumovirus (hMPV), human coronavirus (hCoV) 229E, hCoV NL63, hCoV OC43, parainfluenzavirus (PIV)-1,

PIV-2, PIV-3, PIV-4, RV, human enterovirus, and human bocavirus] and bacterial respiratory pathogens (Streptococcus pneumoniae, Hemophilus influenzae, Chlamydophila pneumoniae, Legionella pneumophila, Bordetella pertussis, Bordetella parapertussis, and Mycoplasma pneumoniae) using the AllplexTM respiratory panel assay (Seegene, Korea).

- Climatic factors was analyzed.

- The AllplexTM gastrointestinal panel assay (Seegene, Korea) was used to detect viral (rotavirus, NoV-I, NoV-II, astrovirus, enteric adenovirus, sapovirus), bacterial [Salmonella spp., Shigella spp., Vibrio spp., Campylobacter spp., Clostridium difficile toxin B, Clostridium perfringens, Yersinia enterocolitica, E. Coli O157:H7, enterohemorrhagic E. Coli (stx1/2), enteropathogenic E. Coli (eaeA), ETEC (It/st), EAEC (aggR), Aeromonas spp.], and protozoan (Giardia lamblia, Cryptosporidium spp.,

Blastocystis hominis, Dientamoeba fragilis,

Cyclospora cayetanensis) pathogens.

- Conventional RT-PCR for HPeV, klassevirus, NoV-GIV, and Aichivirus was performed.

- Semi-nested PCR was performed to confirm the genotypes of HRV, NoV, GIl, and HPeV

\section{Results} March 8, April 22, April 23, April 24, and April 27)

- During the Asian dust period, ambient air samples were collected at Yangjae (six samples), Pyeongchang (one sample), and Jayang (one sample); 63 air samples were collected (Yangjae-26, Jayang-19,

Pyeongchang-18) during the non-Asian dust period.

- The monthly distribution of collected air samples was as follows: 20 samples were collected in March (March 6, 7, 8, 9, 10, 21, 22 , and 30), 18 samples in April (April 22, 23, 24, and 30), 5 samples in May (May 7, 13, 28, and 29), 12 samples in (June 9,11, 21, and 23), 11 samples in July (July 12, 14, 21, and 28), 4 samples in August (August 2 and 4 ), and one sample in September (September 6).

- The daily average concentrations of PM10 were 52.1 and $44.8 \mu \mathrm{g} / \mathrm{m} 3$ in the Asian and nonAsian dust periods

- The daily average concentrations of PM2.5 were 51.4 and $44.5 \mu \mathrm{g} / \mathrm{m} 3$ in the Asian and non-Asian dust periods.

- HRV was detected in ambient air samples collected during the non-Asian dust period on April 7 (Yangjae), June 9 (Yang Jae), June 23 (Jongro), and July 21 (Yangjae), 2016.

- One air sample collected during the Asian dust period on April 23, 2016 (Table 1) was positive for HRV
- Asian dust was observed for 6 days (March 6

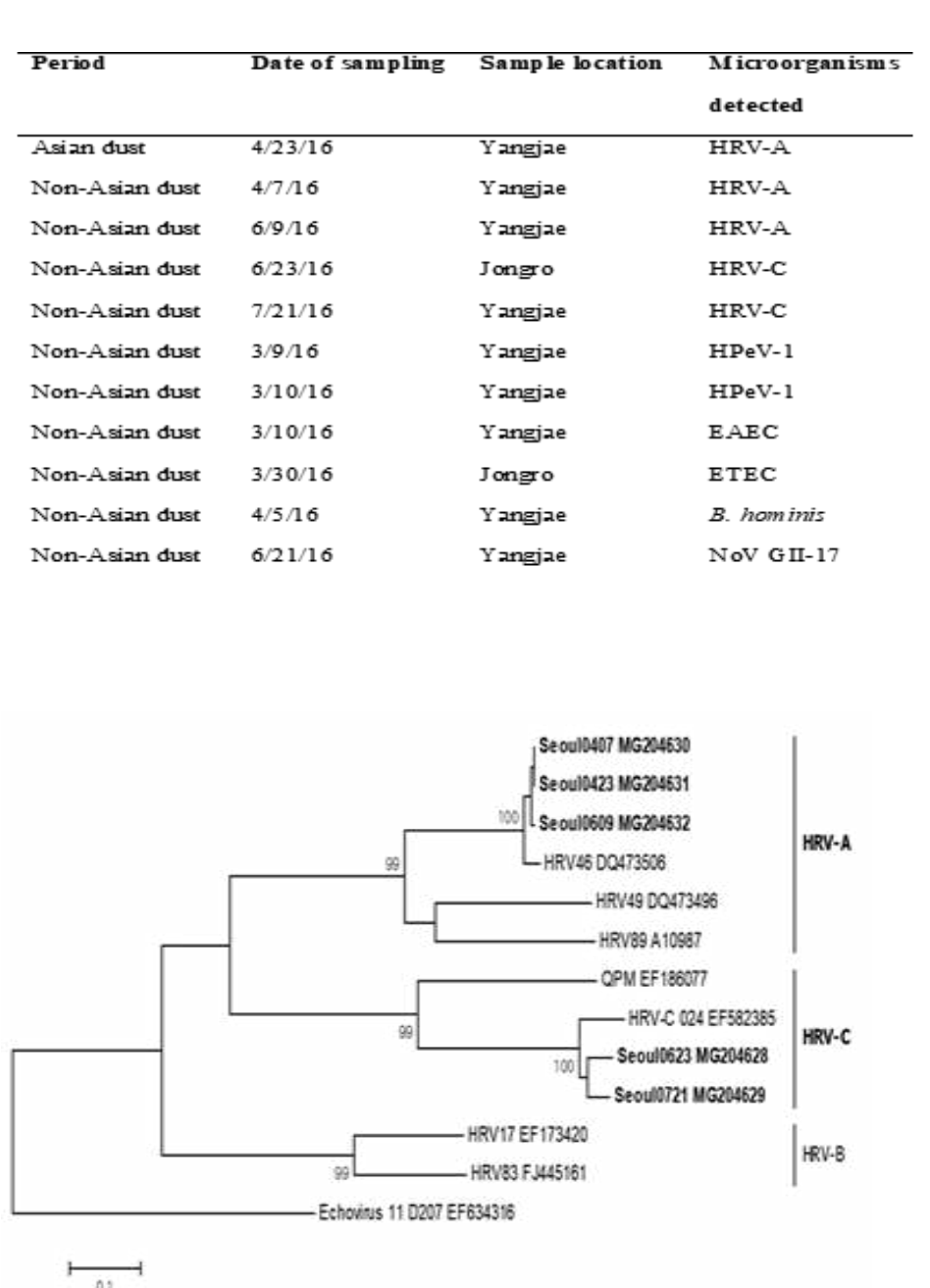

Fig. 1 Phylogenetic analysis of the partia VP4/VP2 (-440 bp) gene of HRVs. The bootstrap values from 1,000 replicates are shown on each branch.

- RT-PCR was negative for the presence of klassevirus, Aichivirus, and NoV GIV in all samples collected during Asian dust period and non-Asian dust period.

HPeV was detected in two air samples collected during the non-Asian dust period on March 9 (Yangjae) and March 10 (Yangjae), 2016; they were determined to be HPeV-1.
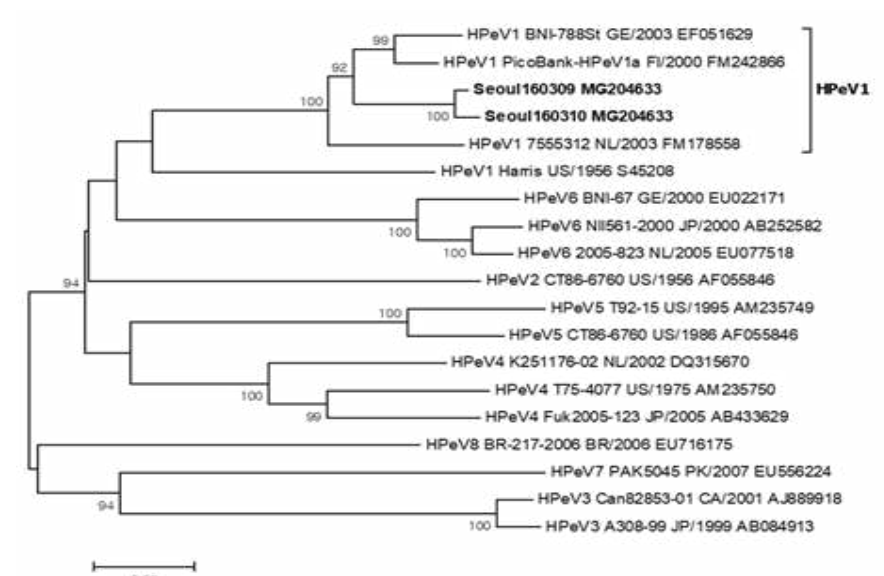

Fig. 2 Phylogenetic analysis of the partial VP1 (741 bp) gene of HPeVs.

- A sample collected on June 212016 (Yangjae) was positive for NoV, and it had the NoV Gll-17 genotype (Fig. 3)

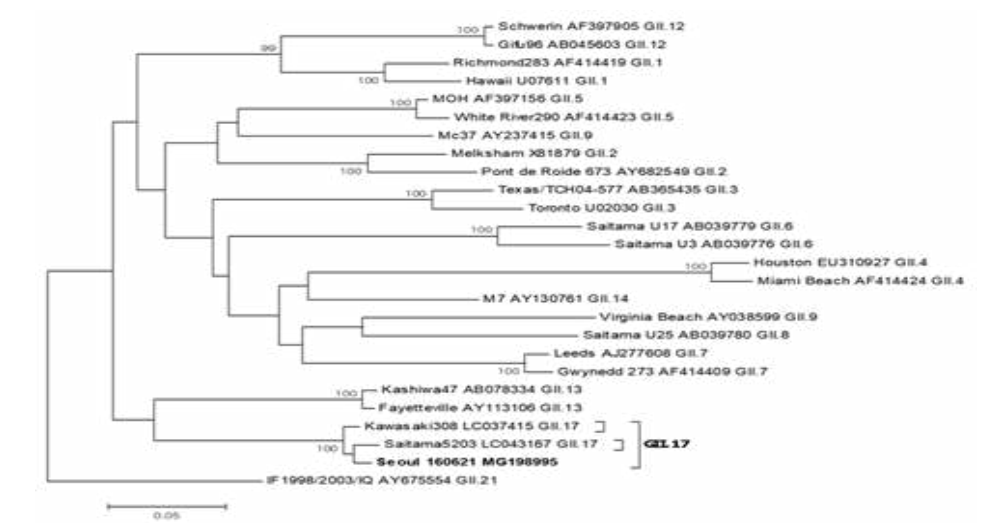

Fig. 3 Phylogenetic analysis of the partial capsid sequence (303 bp) of Gll NoV.

\section{Conclusions}

- This study was the first to confirm the presence of pathogenic viruses, including $\mathrm{HRV}, \mathrm{NoV}$, and HPeV in ambient air in Korea.

- Most of these pathogenic viruses were detected in ambient air samples during the non-Asian dust period, which suggests that Asian dust might not play a major role in epidemics caused by viral pathogens, although further studies are needed to confirm these findings. 